\title{
Telehealth Physical Therapy: More Than an Alternative to In Person Care of the Young Athlete
}

\author{
Joseph T. Molony Jr., PT, MS, SCS, $\operatorname{CSCS}^{1}$ a \\ 1 Young Athlete Program, Hospital for Special Surgery \\ Keywords: young athlete, telehealth, physical therapy, rehabilitation, pediatric, adolescent \\ https://doi.org/10.26603/001c.29511
}

\section{International Journal of Sports Physical Therapy}

Vol. 16, Issue 6, 2021

\begin{abstract}
The shift to telehealth due to COVID-19 revealed that a new care model for the young athlete, which combines in-person and virtual visits, could be an enhancement to in-person care alone. This clinical suggestion is novel as it discusses the utility of a hybrid care model for the young athlete, which has not yet been described. Interacting with the patient and family virtually in the home environment offers benefits that are difficult to achieve in the clinic. Opportunities such as the ability to custom tailor the home program with consideration of the patient's learning abilities, provide movement quality feedback outside of the clinical environment, observe parent/caregiver feedback, involve family members who may not be available to attend in-person visits, and the possibility of converting an in-person cancelation to a telehealth visit in order to maintain continuity of care, are examples of how this model may optimize treatment. Consideration of investigating the impact on clinical outcomes and cost effectiveness is recommended.
\end{abstract}

\section{Level of Evidence}

5

\section{THE PROBLEM}

Integration of a home exercise program is a vital component to a successful outcome when treating the young athlete, however, ensuring accurate performance is difficult. Without the ability to observe the patient in the home environment and provide real-time instruction to him/her and parents/caregivers it is challenging to determine if the program is being properly implemented. Home exercise program technique out of the clinical setting may fall short without the watchful eye of a clinician. Parents/caregivers independently attempting to provide proper guidance and feedback regarding the program in the home environment may not be effective. When in the clinic, the PT asking the patient to demonstrate their program, with and without parent/caregiver feedback, may not be sufficient to achieve the same results when back at home.

\section{THE SOLUTION}

Shelter in place due to COVID-19 prompted a rapid shift in the profession of physical therapy from in-person visits to telehealth. ${ }^{1,2}$ In the young athlete population this presented an opportunity to provide care using a new practice model where the patient could be visualized and treated in the home environment virtually with his/her parents/caregivers. This shift has revealed that telehealth can be a powerful tool which allows observation of movement outside of the clinic, instruction of parents/caregivers regarding feedback on movement quality while at home, the development of a custom-tailored home program with consideration of the patient's learning abilities and home setting, and alternative scheduling opportunities, all of which may enhance outcomes and reduce healthcare costs in this population. This process can assist in mitigating the problem of ineffective home program performance due to improper exercise technique, which can have a detrimental impact on recovery. This clinical suggestion is novel in that it discusses the

\footnotetext{
a Corresponding Author:

Joseph T. Molony

HSS/Hospital for Special Surgery

Pediatric Rehabilitation, 9th floor

535 East 70th Street, New York, NY 10021

Phone: 212 606-1137

Fax: 212 774-2761

Email: molonyj@hss.edu
} 
utility of a hybrid care model specifically for the young athlete, which has not yet been described. ${ }^{1,3,4}$

One advantage of telehealth as an adjunct to in-person care of the young athlete is the ability to observe patient movement patterns outside of the clinical environment. The clinic is an ideal setting for performance of exercises and functional movement, particularly with the therapist physically present providing hands on facilitation of proper technique. At home through a virtual visit the young athlete is more likely to demonstrate the movement patterns they would typically perform when not in the direct presence of the therapist. Additionally, telehealth provides the potential to assess the young athlete in his/her sport specific setting. This can be outdoors or indoors such as on a court surface or on the ice.

Another benefit of virtual visits for this population is education of the parent/caregiver regarding observation of movement and how to instruct the patient on proper exercise technique, as it is possible that learning in the clinic may not translate completely to accurate performance of these skills at home. Accurate performance of the young athlete's treatment plan in the home environment is challenging to identify in the clinic, where therapists have the ideal equipment, space, and surfaces to perform tasks. Physical therapists may discover through telehealth that the implementation of the program at home is not as intended and this inconsistency with our instruction may not be recognized by the patient and parents/caregivers. With this discovery physical therapists are positioned to educate the patient and parents/caregivers more accurately about the plan and to improve program efficacy. Additionally, parents/caregivers who may not be able to attend clinic-based visits may be available through telehealth to obtain this same learning.

Developing an effective home program for the young athlete is very challenging as there are multiple factors to consider:

- Is the patient able to think abstractly and adapt the program to the home environment?

- Does the patient have any learning impairments? Though he or she may not be receiving special education, that does not necessarily mean that there are no barriers to learning.

- Does the patient truly understand the exercises and their intended purpose?

- What is the patient's ability to provide effective feedback to the therapist or parents/caregivers regarding muscle fatigue versus pain?

- To what extent is the parent or caregiver able to provide guidance regarding the details of the exercises, such as the proper movement patterns, alignment, and pace?

- What will be the ideal choice and number of exercises to optimize program adherence?

- Are there appropriate locations in the home to perform the various exercises?

- Does the patient have access to any exercise equipment? If so, what items? If there is access to a school or fitness facility how likely is it that the patient will go due to time constraints or transportation logistics?
While these factors can be addressed to some extent in the clinic, telehealth provides the opportunity to optimize home program implementation. Being able to see the young athlete in the home environment provides the therapist with a wealth of information that is difficult for the family to convey during in-person care and challenging to obtain through discussion. The therapist's ability to see such things as the amount of space for exercise, height of stairs, type of flooring, exercise equipment, household items available, exercise technique etc. enables him/her to accurately instruct the patient in home program performance. For example, an injured adolescent athlete who was instructed to advance single limb balance to an unstable surface at home to mimic a foam balance pad in the clinic, stood on a pillow. In observing the patient during a telehealth visit this was no more challenging than standing on a flat surface. It became evident that the pillow was fully compressed and not providing the intended dynamic base of support. A change was made to a firmer pillow and the intended balance challenge was achieved. Had it not been for telehealth, this patient would likely have been standing on a compressed pillow, day in and out, not obtaining the neuromuscular benefit of the exercise. Other exercises in the program would perhaps also be misinterpreted, and the duration of recovery potentially unnecessarily extended. Periodic telehealth visits as part of a rehabilitation program for this population provide the opportunity for in home instruction which may be superior to traditional all in-person care.

Telehealth also augments rehabilitation of the young athlete population by providing additional scheduling options which help to ensure continuity of care. Busy family life makes attendance unpredictable and most youth are dependent upon others for transportation. It is not only the lives of the patients which affects their ability to travel to a visit, but the lives of their parents/caregivers and siblings as well. Changes in schedules of a brother or sister and unexpected work obligations of parents/caregivers can cause a cancelation. When a family may not be able to travel to an appointment, they may be able to keep their visit via conversion to telehealth, preventing an interruption in care. Telehealth also provides the opportunity for a brief visit to review and update a home program when adjustments are indicated but a full visit is unlikely to be required. A family may be hesitant to travel for a brief visit in-person, but happy to attend a brief visit via telehealth. Both advantages keep the patient and family engaged without interruptions in treatment, optimizing the episode of care, possibly maximizing the pace of recovery, and in the case of a brief visit, also provide a direct cost reduction to insurers.

\section{DISCUSSION}

While virtual visits cannot completely replace in-person treatment, consideration should be given to a new practice model for care of the young athlete where telehealth is provided in conjunction with in-person visits. Telehealth does not simply need to be an alternative to in-person care, and can be utilized in a "hybrid" model. The ability to observe movement patterns in the patient's home environment and sports setting, to instruct parents/guardians in 
exercise guidance while at home, to custom tailor the home program to the patient's learning abilities and access to exercise equipment/substitute household items, and to provide additional scheduling options, all contribute to a potentially enhanced treatment process. This process may mitigate some of the problems related to ineffective home program performance in the young athlete population, which can have a detrimental impact on recovery. There is a potential cost savings associated with this model if it yields a more rapid recovery and requires fewer skilled visits. This could incentivize insurers to cover telehealth services as an adjunct to in-person care. Investigation of patient outcomes, ideal number and timing of telehealth visits in an episode of care, and cost effectiveness of this blended model compared to in-person alone would be a productive next step. ${ }^{5}$

\section{DISCLOSURES}

The author reports no relevant disclosures.

Submitted: April 10, 2021 CST, Accepted: September 19, 2021

CST 


\section{REFERENCES}

1. Miller MJ, Pak SS, Keller DR, Barnes DE. Evaluation of pragmatic telehealth physical therapy implementation during the COVID-19 pandemic. Phys Ther. 2021;101(1). doi:10.1093/pti/pzaa193

2. Cottrell MA, Russell TG. Telehealth for musculoskeletal physiotherapy. Musculoskelet Sci Prac. 2020;48(102193). doi:10.1016/j.msksp.2020.102 193

3. Turolla A, Rossettini G, Viceconti A, et al. Musculoskeletal physical therapy during the COVID-19 pandemic: Is telerehabilitation the answer? Phys Ther. 2020;100(8):1260-1264.
4. Galea MD. Telemedicine in rehabilitation. Phys Med Rehabil Clin N Am. 2019;30(2):473-483. doi:10.1016/ j.pmr.2018.12.002

5. Lee AC, Davenport TE, Randall K. Telehealth physical therapy in musculoskeletal practice. J Orthop Sports Phys Ther. 2018;48(10):736-739. doi:10.2519/jo spt.2018.0613 